\title{
Portal Vein Embolization before Right Hepatectomy: Improved Results Using n-Butyl-Cyanoacrylate Compared to Microparticles Plus Coils
}

\author{
Boris Guiu • Pierre Bize - Daniel Gunthern • \\ Nicolas Demartines $\cdot$ Nermin Halkic • \\ Alban Denys
}

Received: 12 November 2012/ Accepted: 26 December 2012/Published online: 30 January 2013

(C) Springer Science+Business Media New York and the Cardiovascular and Interventional Radiological Society of Europe (CIRSE) 2013

\begin{abstract}
Background There is currently no consensus in the literature on which embolic agent induces the greatest degree of liver hypertrophy after portal vein embolization (PVE). Only experimental results in a pig model have demonstrated an advantage of n-butyl-cyanoacrylate (NBCA) over 3 other embolic materials (hydrophilic gel, small and large polyvinyl alcohol particles) for PVE. Therefore, the aim of this human study was to retrospectively compare the results of PVE using NBCA with those using spherical microparticles plus coils.

Methods A total of 34 patients underwent PVE using either NBCA $(n=20)$, or spherical microparticles plus coils $(n=14)$. PVE was decided according to preoperative volumetry on the basis of contrast-enhanced CT. Groups were compared for age, sex, volume of the left lobe before PVE and future remnant liver ratio (FRL) (volume of the left lobe/total liver volume - tumor volume). The primary end point was the increase in left lobe volume 1 month after PVE. Secondary end points were procedure complications and biological tolerance.

Results Both groups were similar in terms of age, sex ratio, left lobe volume, and FRL before PVE. NBCA induced a greater increase in volume after PVE than did microparticles plus coils (respectively, $+74 \pm 69 \%$ and $+23 \pm 14 \%$,
\end{abstract}

B. Guiu $(\bowtie) \cdot$ P. Bize · D. Gunthern · A. Denys

Digestive \& Oncologic Imaging and Interventional Radiology

Unit, Department of Radiology and Interventional Radiology,

Centre Hospitalo-Univeristaire Vaudois, Rue du Bugnon 46,

1011 Lausanne, Switzerland

e-mail: boris.guiu@chu-dijon.fr

N. Demartines · N. Halkic

Department of Visceral Surgery, Centre Hospitalo-Univeristaire

Vaudois, Lausanne, Switzerland $p<0.05$ ). The amount of contrast medium used for the procedure was significantly larger when microparticles and coils rather than NBCA were used (respectively, $264 \pm 43 \mathrm{ml}$ and $162 \pm 34 \mathrm{ml}, p<0.01$ ). The rate of PVE complications as well as the biological tolerance was similar in both groups. Conclusion NBCA seems more effective than spherical microparticles plus coils to induce left-lobe hypertrophy.

Keywords Comparative study - Embolic material . Embolization - Liver regeneration · Portal vein

\section{Introduction}

Since the first report of portal-branch ligation to induce hypertrophy of the contralateral liver lobe in rabbits in 1920 [1] and the first use of portal vein embolization (PVE) in clinical practice in 1986 by Kinoshita et al. [2], many techniques of portal vein occlusion have been described [3]. Various embolic materials have been proposed for the complete occlusion of the right portal branches both proximally and distally thereby preventing recanalization of the portal vein or collateralization from the left side $[4,5]$. American studies [6] have mainly reported embolization using spherical particles associated with proximal coils, while European groups mainly use n-butyl-cyanoacrylate (NBCA) mixed with iodized oil [7, 8]. Experimental results in a pig model [9] have demonstrated the advantage of NBCA over three other embolic materials (hydrophilic gel, and small and large polyvinyl alcohol particles), the second best embolic material being small $(50-150 \mu \mathrm{m})$ particles. However, to our knowledge, this result has never been confirmed in a human study. Thus, the objective of this study was to compare two consecutive groups of patients selected according to the same criteria 
by the same surgical and radiological team, and embolized with either NBCA or microparticles plus coils.

\section{Materials and Methods}

This study consisted of a retrospective analysis of two consecutive groups of patients. All patients gave their written informed consent for the procedure and our local ethics committee approved the retrospective analysis of their data.

All consecutive patients selected for right PVE were included in this study. Between January 2010 and October 2010, the first 14 patients, with a median age of 69 (range 52-74) years, underwent right PVE using spherical microparticles plus coils as described by Madoff and coll. [10]. Five patients had HCC developed on liver cirrhosis, while eight had liver metastases and one had intrahepatic cholangiocarcinoma (Table 1). Between November 2010 and January 2012, the next 20 patients, with a median age of 68.5 (range 46-79) years, underwent right PVE using NBCA. Seven patients had hepatocellular carcinoma (HCC) developed from liver cirrhosis, while 13 patients had liver metastases (Table 1). No patient had undergone prior therapy such as transarterial chemoembolization or systemic chemotherapy.

\section{Inclusion and Exclusion Criteria}

All of the patients gave their written informed consent. Patients with liver metastases developed on a healthy liver were considered for right PVE if the planned resection would have left less than $30 \%$ of functional liver parenchyma estimated on liver volumetry measured by CT before surgery. All of the patients with hepatocellular carcinoma developed on cirrhosis were considered for PVE if right hepatectomy was planned. Patients with HCC developed on cirrhosis were eligible for right hepatectomy if the $\mathrm{HCC}$ was located in the right lobe, if they had Child Pugh grade A cirrhosis, if there was no evidence of extrahepatic disease, if the portal and hepatic veins and bile ducts were patent, and if they were less than 75 years-old. In our center, PVE was not considered if patients had extrahepatic tumors, Child-Pugh grade B or C cirrhosis, trans-arterial chemoembolization in the previous 3 months, signs of active alcoholic hepatitis, serum alanine aminotransferase levels more than twice the normal values [11], or if they were being treated with interferon therapy. Patients considered for embolization of the right portal branches plus segment IV branches were not included in this study.

The decision to treat (right PVE and liver resection) was taken at the weekly liver tumor board meeting. The board included liver surgeons, oncologists, hepatologists, and interventional radiologists. For all of the patients included in this study right hepatectomy for the removal of segments V, VI, VII and VIII had been planned.

\section{Technique of PVE}

PVE was performed under general anesthesia by an interventional radiologist with 15 years of experience in interventional liver procedures, during a $48 \mathrm{~h}$ hospital stay. PVE using NBCA was performed according to the technique previously described by De Baere et al. [7]: A left portal vein branch (preferentially a segment III branch) was punctured under sonographic guidance using a $30 \mathrm{~cm}$ long $5 \mathrm{~F}$ Ring needle catheter (Cook, Bloomington, IN, USA) and portal pressure was measured. The Ring catheter was advanced over a 0.035-inch hydrophilic guide wire (Terumo, Tokyo, Japan) into the portal trunk where portography was performed. The right second-order portal branches were selectively catheterized one by one using the ring catheter and then embolized with boluses of $0.5 \mathrm{ml}$ of a mixture of Lipiodol and n-butyl-cyanoacrylate (Histoacryl, B Braun, Tuttlingen, Germany) after checking that the flow was hepatopetal (a mixture of $1 \mathrm{ml}$ of n-butyl cyanoacrylate with 1-3 ml of iodized oil was used). Dilution of the NCBA and Lipiodol was adapted to the portal venous flow so that both distal and proximal branch occlusion could be achieved. In case of rapid flow (as usually observed at the beginning of the

Table 1 Comparison of baseline characteristics of patients receiving PVE using NBCA or microparticles plus coils

\begin{tabular}{llll}
\hline Characteristic & $\begin{array}{l}\text { NBCA } \\
(n=20)\end{array}$ & $\begin{array}{l}\text { Microparticles plus coils } \\
(n=14)\end{array}$ & $>0.05$ \\
\hline Age, mean \pm SD & $68 \pm 12$ & $67 \pm 9$ & $>0.05$ \\
Sex ratio, F/M & $6 / 14$ & $5 / 9$ & $>0.05$ \\
Cirrhosis/metastases & $7 / 13$ & $5 / 9$ & $>0.05$ \\
Total liver volume, ml, mean \pm SD & $1978 \pm 1352$ & $1692 \pm 391$ & $>0.05$ \\
Left lobe volume, ml, mean \pm SD & $470 \pm 210$ & $495 \pm 191$ & $>0.05$ \\
FRL ratio, mean \pm SD & $0.27 \pm 0.11$ & $0.29 \pm 0.06$ & \\
\hline
\end{tabular}

$P V E$ portal vein embolization, NBCA n-butyl-cyanoacrylate, $S D$ standard deviation, $F R L$ future remnant liver 
embolization), dilution with a ratio of NCBA/lipiodol of 1:2 was used. In case of reduced portal flow, a dilution of 1:1 was used to avoid the migration of the embolic agent into the left branches. To reduce this risk, NBCA was injected selectively into the segmental branches. After embolization, the Ring catheter was carefully flushed in the right portal branch with $5 \%$ glucose solution and then repositioned in the portal trunk, where post-PVE portography was performed and portal pressure was measured.

PVE using microparticles plus coils was derived from the technique described by Madoff et al. [6]. We decided to use small spherical particles after the results of an experimental study in pig [9]. We used the same portal approach and the same Ring catheter as for NBCA PVE. Embolization was achieved with 100-300 $\mu \mathrm{m}$ spherical particles injected selectively into the distal portal branches (Bead Block, Terumo, Japan). A 1:1 dilution was used between particles and contrast media as recommended by Terumo. Once stasis had been achieved with microparticles in the peripheral branches, 0.035-inch coils (Tornado; Cook) were also placed proximally in order to occlude the origin of the right portal branches. For all procedures, portal pressure was measured before and at the end of the embolization procedure. The amount of contrast medium injected was recorded for each procedure.

\section{Volumetric CT Assessment and Outcome Evaluation}

Baseline computed tomography (CT) imaging data were obtained a mean of 8 (range 1-21) days before PVE, and follow-up imaging was performed a mean of 30 (range 25-35) days after PVE. CT images with a $2.5 \mathrm{~mm}$ slice thickness were obtained during the portal phase (70 s after injection of $100 \mathrm{ml}$ of $350 \mu \mathrm{g} / \mathrm{ml}$ iodine at $3 \mathrm{ml} / \mathrm{s}$ ) after injection of contrast media with a 16-detector row Lightspeed multislice CT unit (General Electric, Milwaukee, WI, USA) as previously described $[12,13]$. On each image, the whole liver, the tumor and the left lobe (segments II, III, and IV) were delineated with a handheld cursor [14] and their respective volumes were automatically calculated by the workstation (Advantage Windows; General Electric). Depending on the vascular anatomy of the liver, the delineation of the right and left lobes was defined by the plane between the gallbladder fossa and the middle hepatic vein to the inferior vena cava. Total functional liver volume (TLV) was defined as the total liver volume minus the tumor volume. The ratio between the left lobe volume and the TLV was then calculated and defined as the future remnant liver ratio (FRL).

\section{Biological Changes after PVE}

Serum liver enzymes were assessed by measuring serum aspartate amino transferase (AST), prothrombin time (PT) and total bilirubin levels before and 1 month after PVE.
Study End Points

The primary end point of the study was to compare left lobe volume and FRL increase after PVE using microparticles plus coils vs. NBCA. Secondary end points were comparison of patient outcome, biological changes and portal pressure modifications after PVE in the two groups. We also compared the amount of embolic material necessary to obtain PVE in metastatic vs. HCC patients in each group. Finally, we compared the amount of contrast medium used for the procedures.

\section{Statistical Analysis}

The volumetric results are reported as means \pm standard deviations unless stated otherwise. The normal distribution of variables was checked using the Shapiro-Wilk test. When variables did not have a normal distribution, a MannWhitney test was applied for comparison. Comparisons of FRL and left liver volumes, portal pressures, and laboratory tests before and 1 month after PVE were performed by a paired $t$ test. Comparison between the two groups was performed by Fisher's test and an unpaired $t$ test. A $p$ value of $<0.05$ was considered statistically significant.

Statistical analysis was performed by JMP software (SAS Institute, Cary, NC, USA).

\section{Results}

The groups receiving NBCA or spherical particles were similar in terms of age, sex ratio, the proportion of cirrhotic patients, left lobe volume and FRL before PVE (Table 1).

PVE was achieved in all patients. No complication related to the procedure was observed during this study in either group. At the 1 month follow-up CT, imaging revealed that one patient had small fragments of NBCA in distal left portal branches without compromising the patency of proximal and segmental portal vein branches.

Embolization using microparticles required a mean number of $10.3 \pm 3.4$ vials of 100-300 $\mu$ m particles. In HCC patients, the amount of embolic material per patient was significantly less than that necessary to achieve PVE in metastatic patients ( $7.6 \pm 2.7$ vs. $11.77 \pm 2.9$ vials, respectively, $p<0.05)$. The number of coils per patient was similar in these two categories of patients. The amount of contrast medium used for the procedure was $264 \pm 43 \mathrm{ml}$ and $162 \pm 34 \mathrm{ml}$, for microparticles plus coils and NBCA, respectively $(p<0.01)$. No renal dysfunction was observed in either group.

\section{Volumetric Efficacy}

After PVE, the left lobe increased in size from $495 \pm 191 \mathrm{ml}$ to $600 \pm 198 \mathrm{ml}$ with microparticles plus coils and from 
$470 \pm 210 \mathrm{ml}$ to $682 \pm 173 \mathrm{ml}$ with $\mathrm{NBCA}$, leading to a left lobe increase in size of $23 \pm 14 \%$ and $74 \pm 69 \%$ $(p=0.046)$, respectively (Figs. 1, 2,3). The increase in the FRL ratio was $29 \pm 16 \%$ and $48 \pm 36 \%$ for patients embolized with microparticles plus coils and NBCA, respectively $(p=0.07)$.

\section{Biological Changes, Portal Pressures, and Patient Outcome}

Serum levels of liver enzymes did not significantly differ before and 1 month after PVE. No significant differences were found between the two groups (Table 2). The increase in portal pressure measured after PVE did not significantly differ in the group microparticles plus coils vs. NBCA: $2.9 \pm 1.3 \mathrm{~mm} \mathrm{Hg}$ and $3.2 \pm 1.2 \mathrm{~mm} \mathrm{Hg}$, respectively.

In the NBCA group, $15(75 \%)$ of the 20 patients underwent right hepatectomy. Three patients had tumor progression, which precluded resection. One HCC patients developed extrahepatic metastases that were not visible on the first evaluation, and during the operation one patient was found to have peritoneal carcinomatosis. In the

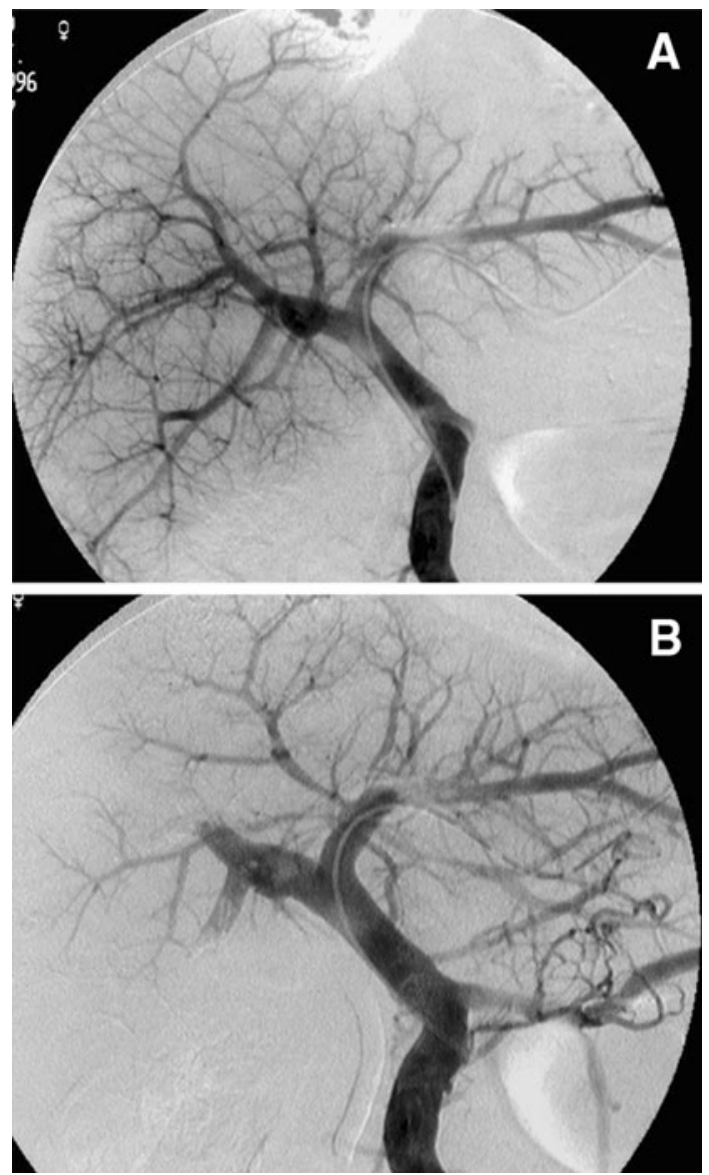

Fig. 1 Portography before (A) and after (B) PVE using NBCA in a 64-year-old patient with HCC microparticles plus coils group, $11(78.6 \%)$ patients underwent surgical resection, 10 by right hepatectomy and one by multiple nonanatomic resections. Three patients were not operated on: one HCC patient because of additional nodules found in the left lobe; one HCC patient with ChildPugh A cirrhosis because of an insufficient increase in left lobe volume after PVE; and one patient because of peritoneal extension of the sigmoid cancer discovered during laparotomy.
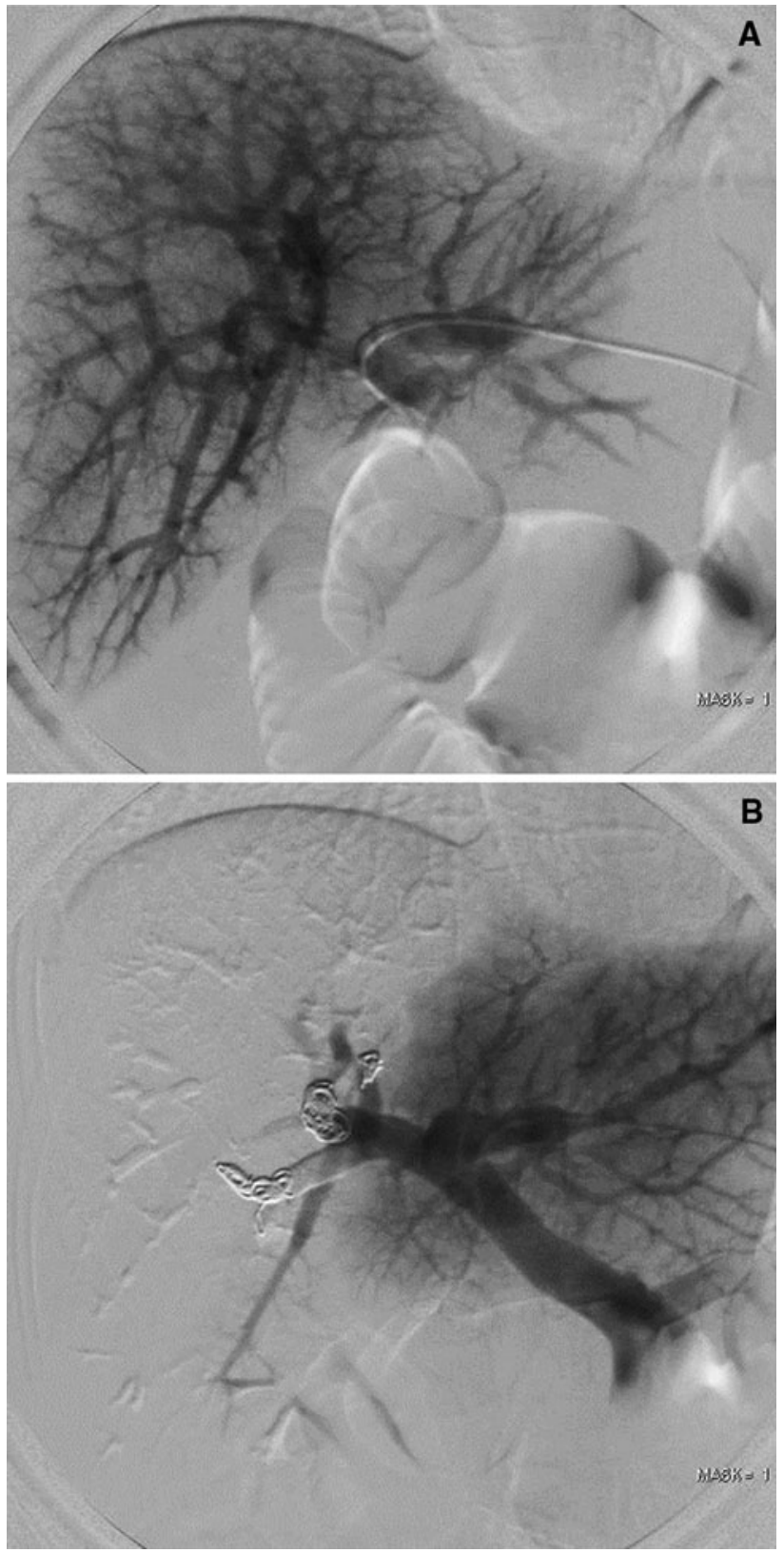

Fig. 2 Portography before (A) and after (B) PVE using microparticles plus coils in a 73-year-old patient with liver metastases from sigmoid cancer 


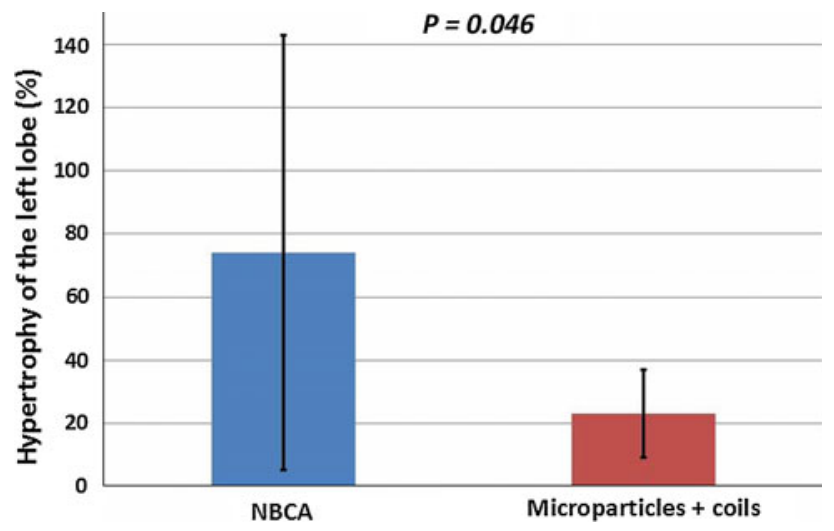

Fig. 3 Graph showing the mean \pm SD increase in left lobe volume 1 month after PVE using NBCA and microparticles plus coils

\section{Discussion}

Different embolic materials have been proposed for PVE. In the initial study of Makuushi et al., a gelatin sponge mixed with diatrizoate sodium meglumine and gentamycin was used [15]. However, frequent recanalizations were observed after embolization, and these required additional procedures. Ogasawara et al. reported the use of absolute alcohol injected through an occlusion balloon into the right lobe [16]. This technique seemed very efficient in terms of hypertrophy, but was poorly tolerated especially in patients with chronic liver disease in whom major changes in liver function may preclude surgery. Finally, most American and European teams use either a combination of microparticles plus coils [6] or a mixture of NBCA and iodized oil [7, 8, 17]. Although these two techniques have produced good results in clinical practice and may seem similar in terms of results and complications [18], they are difficult to compare using data from the literature. As recently reported in a meta-analysis, it is impossible to determine the best embolic agent for PVE [3]. Series cannot be compared easily because of different case mixes of liver metastases [6, 19] and hepatocellular carcinoma $[8,20,21]$, because of different delays (from 2 weeks [20] to 5 weeks [8]) for the volumetric assessment, or even because patients received associated techniques like chemoembolization, which affects regeneration [22]. More importantly, the degree of hypertrophy of the FRL was not measured in the same way in the different series [23]. Indeed, some authors assessed hypertrophy by comparing the ratio of the left lobe volume divided by the total measured liver volume minus the tumor volume before and 1 month after PVE. However, this volumetric end point may be influenced by an increase in left lobe volume, by a decrease in embolized liver volume, or by both mechanisms. Authors who used microparticles plus coils divided the left lobe volume by an estimated total liver volume. This estimation was derived from a formula based on body surface area to give a theoretical total liver volume [12], which although simple to obtain, cannot reflect true liver volume in all cases.

Therefore, we decided to compare different embolic agents for PVE both experimentally and clinically in reproducible conditions. The present study demonstrated that a greater degree of liver hypertrophy was achieved with NBCA than with microparticles plus coils. This result is in keeping with the results observed in an experimental work [9] comparing various embolic materials for PVE in a pig model. In this animal study, hypertrophy of the nonembolized liver was evaluated histologically and on the basis of liver lobe surface area measurements. It was demonstrated that NBCA was better able to induce liver hypertrophy than were small $50-150 \mu \mathrm{m}$ nonspherical particles, which in turn were better than large $(500-700 \mu \mathrm{m})$ spherical particles. Because it has been reported that very small particles, below $100 \mu \mathrm{m}$, are dangerous for use in arterial embolization of the liver because they might induce fatal pulmonary embolization of small distal pulmonary arteries [24], we decided to use 100-300 $\mu \mathrm{m}$ spherical particles in our clinical study. With this size of particles, no pulmonary complications after PVE have ever been reported [3]. In the experimental study in pig, the ratio of surface areas of liver lobules in the nonembolized vs. embolized liver was $40 \%$ higher for NBCA than for small particles. We found a similar picture in this clinical study in terms of FRL

Table 2 Biological tests before PVE and surgery in the NBCA group and in the microparticles plus coils group

\begin{tabular}{llll}
\hline Characteristic & NBCA, mean \pm SD & Microparticles plus coils, mean \pm SD & $p$ \\
\hline Total bilirubin before PVE, $\mu \mathrm{mol} / \mathrm{L}$ & $12 \pm 4$ & $12 \pm 4$ & $>0.05$ \\
Total bilirubin before surgery, $\mu \mathrm{mol} / \mathrm{L}$ & $13 \pm 5$ & $13 \pm 3$ & $>0.05$ \\
AST before PVE, U/L & $34 \pm 12$ & $31 \pm 13$ & $>0.05$ \\
AST before Surgery, U/L & $36 \pm 13$ & $32 \pm 14$ & $>0.05$ \\
PT before PVE, U/L & $88 \pm 7$ & $90 \pm 6$ & $>0.05$ \\
PT before surgery, U/L & $86 \pm 9$ & $87 \pm 7$ & $>0.05$ \\
\hline
\end{tabular}

In both groups, total bilirubin, AST, and PT values before PVE did not significantly differ from their values before surgery

$P V E$ portal vein embolization, $N B C A$ n-butyl-cyanoacrylate, $S D$ standard deviation, $A S T$ aspartate aminotransferase, $P T$ prothrombin time 
hypertrophy measured by CT volumetry when NBCA was compared with spherical 100-300 $\mu \mathrm{m}$ particles plus coils. One explanation for the better results of NBCA could be the inflammatory reaction observed in the periportal area as mentioned in a previous publication [7] as well as in the animal study [9]. Indeed, the present study strongly suggests that the mechanical effect of portal precapillary occlusion is probably not the only mechanism involved in the regeneration phenomenon as suggested by complete right portal vein occlusion and a similar increase in portal vein pressure after PVE in both groups.

We found no differences between the two groups in terms of resection rate after PVE. Our resection rate of $76 \%$ is close to the resection rate of $85 \%$ reported by Abulkhir et al. in a meta-analysis of 1088 patients [3]. We encountered neither renal dysfunction nor side effects related to the contrast medium, but it is noteworthy that we used almost twice as much contrast medium with microparticle plus coil embolization than with NBCA embolization. This may have a potential impact mainly in patients with HCC developed on cirrhosis because these patients are more likely to develop hepatorenal syndrome. Although we did not specifically assess the cost of the two procedures, PVE using large quantities of microparticles together with the coils is likely to be more costly than PVE using only NBCA.

This study has several limitations, including its retrospective design, the small number of patients in each group, and the use of historical controls [25] rather than a randomized trial. However, the baseline characteristics of the groups did not significantly differ, and the statistically significant difference in the increase in left lobe volume confirmed the histological results observed in a pig model.

In conclusion, the results of this clinical series confirm the results obtained in our own experimental study. Although both techniques induced complete occlusion of the portal branches, NBCA seems to induce a greater degree of liver hypertrophy. The periportal inflammation observed with NBCA in the experimental study seems to be an important factor for liver regeneration.

Acknowledgments The authors thank Philip Bastable for revising the English.

Conflict of interest The authors declare that they have no conflict of interest.

\section{References}

1. Rous P, Larimore L (1920) Relation of portal blood to liver maintenance. J Exp Med 31:609-632

2. Kinoshita H, Sakai K, Hirohashi K et al (1986) Preoperative portal vein embolization for hepatocellular carcinoma. World J Surg 10:803-808
3. Abulkhir A, Limongelli P, Healey AJ et al (2008) Preoperative portal vein embolization for major liver resection: a meta-analysis. Ann Surg 247:49-57

4. Abdalla EK, Hicks ME, Vauthey JN (2001) Portal vein embolization: rationale, technique and future prospects. $\mathrm{Br} \mathrm{J}$ Surg 88:165-175

5. van Lienden KP, van den Esschert JW, de Graaf W et al (In press) Portal vein embolization before liver resection: a systematic review. Cardiovasc Intervent Radiol

6. Madoff DC, Abdalla EK, Gupta S et al (2005) Transhepatic ipsilateral right portal vein embolization extended to segment IV: improving hypertrophy and resection outcomes with spherical particles and coils. J Vasc Interv Radiol 16(2 pt 1):215-225

7. De Baere T, Roche A, Elias D et al (1996) Preoperative portal vein embolization for extension of hepatectomy indications. Hepatology 24:1386-1391

8. Denys A, Lacombe C, Schneider F et al (2005) Portal vein embolization with N-butyl cyanoacrylate before partial hepatectomy in patients with hepatocellular carcinoma and underlying cirrhosis or advanced fibrosis. J Vasc Interv Radiol 16:1667-1674

9. de Baere T, Denys A, Paradis V (2009) Comparison of four embolic materials for portal vein embolization: experimental study in pigs. Eur Radiol 19:1435-1442

10. Madoff DC, Hicks ME, Abdalla EK et al (2003) Portal vein embolization with polyvinyl alcohol particles and coils in preparation for major liver resection for hepatobiliary malignancy: safety and effectiveness-study in 26 patients. Radiology 227 : 251-260

11. Farges O, Malassagne B, Flejou JF et al (1999) Risk of major liver resection in patients with underlying chronic liver disease: a reappraisal. Ann Surg 229:210-215

12. Abdalla EK, Denys A, Chevalier P et al (2004) Total and segmental liver volume variations: implications for liver surgery. Surgery $135: 404-410$

13. Sandrasegaran K, Kwo PW, DiGirolamo D et al (1999) Measurement of liver volume using spiral CT and the curved line and cubic spline algorithms: reproducibility and interobserver variation. Abdom Imaging 24:61-65

14. Soyer P, Roche A, Elias D, Levesque M (1992) Hepatic metastases from colorectal cancer: influence of hepatic volumetric analysis on surgical decision making. Radiology 184:695-697

15. Makuushi M, Le Thai B, Takayasu K et al (1990) Preoperative portal embolization to increase safety of major hepatectomy for hilar bile duct carcinoma: a preliminary report. Surgery 107:521-527

16. Ogasawara K, Uchino J, Une Y, Fujioka Y (1996) Selective portal vein embolization with absolute ethanol induces hepatic hypertrophy and makes more extensive hepatectomy possible. Hepatology 23:338-345

17. Azoulay D, Castaing D, Krissat J et al (2000) Percutaneous portal vein embolization increases the feasibility and safety of major liver resection for hepatocellular carcinoma in injured liver. Ann Surg 232:665-672

18. Di Stefano DR, de Baere T, Denys A et al (2005) Preoperative percutaneous portal vein embolization: evaluation of adverse events in 188 patients. Radiology 234:625-630

19. Azoulay D, Castaing D, Smail A et al (2000) Resection of nonresectable liver metastases from colorectal cancer after percutaneous portal vein embolization. Ann Surg 231:480-486

20. Ji W, Li JS, Li LT et al (2003) Role of preoperative selective portal vein embolization in two-step curative hepatectomy for hepatocellular carcinoma. World J Gastroenterol 9:1702-1706

21. Kinoshita H, Hirohashi K, Kubo S et al (1992) Preoperative portal vein embolization for hepatocellular carcinoma. Nihon Geka Gakkai Zasshi 93:1317-1323

22. Ogata S, Belghiti J, Farges O et al (2006) Sequential arterial and portal vein embolizations before right hepatectomy in patients 
with cirrhosis and hepatocellular carcinoma. Br J Surg 93: 1091-1098

23. Denys A, Prior J, Bize P et al (2012) Portal vein embolization: what do we know? Cardiovasc Intervent Radiol 35: 999-1008
24. Brown KT (2004) Fatal pulmonary complications after arterial embolization with 40-120- micro M tris-acryl gelatin microspheres. J Vasc Interv Radiol 15(2 pt 1):197-200

25. Sacks H, Chalmers TC, Smith H Jr (1982) Randomized versus historical controls for clinical trials. Am J Med 72:233-240 